\title{
CVD GROWTH and CHARACTERIZATION OF 2D TRANSITION METAL DICHALCOGENIDES, $\mathrm{MoS}_{2}$ and $\mathrm{WS}_{2}$
}

\author{
Nihan KOSKU PERKGÖZ ${ }^{1}$ \\ ${ }^{\mathbf{1}}$ Electrical and Electronics Department, Engineering Faculty, Anadolu University, Eskişehir, Turkey
}

\begin{abstract}
Two-dimensional transition metal dichalcogenides (TMDC), specifically molybdenum disulfide and tungsten disulfide have received significant attention as their direct bandgap exhibits a shift from indirect to direct as the layer goes to single molecular thickness (2D). Hence, they have high potential to pave way for novel optoelectronic devices. However, their structural and optical properties are still not completely understood, especially, spatial change of photoluminescence intensities, variations in excitons-trions, and shift in peak wavelengths in these 2D flake structures need further investigation. In this research, after growing TMDCs using chemical vapor deposition technique, in addition to measuring micro Raman and photoluminescence spectra, we performed dark field microscopy measurements and photoluminescence mappings to identify grain boundaries and seeding particles. The results clearly show that the flakes, which look lie single-piece through the optical spectroscopy images, in fact, include grain boundaries, seeds and wrinkles. Photoluminescence maps reveal that emission occurs due to different mechanisms such as excitons and trions, depending on the locations on the flakes where the measurement is performed. Our results show that there are different routes that emission can occur and 2D TMDCs provide a rich variety of alternatives to realize novel photonic devices.
\end{abstract}

Keywords: $\mathrm{MoS}_{2}$, WS2, Two-dimensional materials, Chemical vapor deposition, Photoluminescence

\section{INTRODUCTION}

For high-performance optoelectronic devices and systems, realizing materials with high quantum efficiency is crucial for them to be used in different fields including defense industry, optical communications, security, medical applications and advanced technological applications. Currently, different material systems such as GaN, GaAlAs, InGaAs, InGaAsSb are already being used emphasizing their high quantum yield characteristics in the related technology. On the other hand, optoelectronics market share and its usage areas are continuously increasing with higher performance expectations such as superior efficiency, wider spectral range, flexibility, transparency, less material consumption and CMOS integration [1]. To reach these objectives, the drawbacks of the conventional materials, such as that of Si or GaAs based materials, should be overcome. Novel systems are needed to be developed and innovative structures are required to enhance the overall performance criteria. For novel breakthroughs in this area, it is expected that 2 dimensional (2D) material systems have a potential to provide the key technology. In this research, we have grown and characterized $2 \mathrm{D} \mathrm{MoS}$ and $\mathrm{WS}_{2}$, transition metal dichalcogenides (TMDCs). These materials have potential not only be used in optoelectronic devices but also pave the way for other innovative electronic systems with increased performance and functionality [2]. Additionally, with the successful deposition of these 2D TMDCs, it will be possible to discover their excitonic properties such as losses in carrier transport and exciton life time.

When a material makes a transition from its bulk form into other dimensions, the material characteristics is found to change fundamentally, which points out to a high potential to realize novel devices with extra-ordinary properties [3, 4]. Two-dimensional (2D) materials were first realized with the exfoliation of graphene from graphite in 2004, where this new material showed very high electron mobility, 
mechanical strength, high thermal conductivity and flexibility [5-8]. After this discovery, it is suggested that there are about 500 2D materials that are waiting to be found [9]. However, there are still major issues for such 2D materials to transit from laboratories to manufacturing, firstly due to difficulties in large area-growth [10]. For the case of graphene, specifically, it has the zero bandgap, which makes it difficult to build a device without losing the material's superior properties. Hence, there is an ongoing effort of discovering novel 2D material systems rather with a direct bandgap that can be utilized optoelectronics, microelectronics and energy applications. Within this context, TMDCs, $\mathrm{MX}_{2}(\mathrm{M}=\mathrm{Mo}$, $\mathrm{W} ; \mathrm{X}=\mathrm{S}, \mathrm{Se}, \mathrm{Te}$ ), have started to be studied widely in the recent years [11-14]. Their potential has been shown in optoelectronics [15], valleytronics[16], catalysis [17], microelectronics [18] and batteries [19]. Among these TMDCs, 2D WS 2 has also appealed a special attention both in electronics and photonics [20]. Semiconductor TMDCs are found to possess a direct bandgap when they become single-layered [21]. Hence, both $\mathrm{MoS}_{2}$ and $\mathrm{WS}_{2}$ have direct optical bandgaps, high photoluminescence and additionally very interesting excitonic properties in their heterostructures [22]. For these TMDCs also, scalable, high-quality, large-area growth is still a matter of research [17, 23]. Mechanical exfoliation, chronically the first method in history, is not suitable for large area commercial applications. Similarly, it is not easy to use the methods including ionic intercalation and liquid exfoliation for industry [24, 25]. In this respect, chemical vapor deposition (CVD) presents a high potential to realize large-area and single-layer films [17, 23, 26-28]. Although the principal growth mechanism is still not understood fully, up to present promising results are achieved using CVD [29-33]. In this research study, we utilized CVD to obtain $2 \mathrm{D} \mathrm{MoS} 2$ and $\mathrm{WS}_{2}$ flakes where with CVD growth parameters, we could control the grown films. We characterized our flakes by using use micro Raman, photoluminescence (PL) spectra. Monolayer $\mathrm{MoS}_{2}$ and monolayer $\mathrm{WS}_{2}$ have exciton binding energies of $\sim 0.9 \mathrm{eV}$ and $\sim 0.5 \mathrm{eV}$, respectively where exciton (two charge carriers), trions (three charge carriers) and biexcitons (four charge carriers) can occur due to this strong Coulombic interaction [34]. Hence, in this research, we studied change in PL intensities and peak wavelengths spatially.

\section{EXPERIMENTAL}

Figure 1 shows the experimental setup of the CVD system schematically; the CVD reaction chamber with a movable heating zone is specifically designed to grow 2D materials to achieve both fast cooling and multi temperature zones for different precursors. The quartz boats contain high purity $\mathrm{S}$ powder (99.5\%, Alfa) and $\mathrm{MoO}_{3} / \mathrm{WO}_{3}$ (99.9\%, Sigma-Aldrich). $\mathrm{MoO}_{3}$ and $\mathrm{WO}_{3}$ precursors are used to grow $\mathrm{MoS}_{2}$ and $\mathrm{WS}_{2}$, respectively. These precursors are positioned at different temperature zones, which are identified with respect to their melting temperatures, $\sim 820^{\circ} \mathrm{C}$ for $\mathrm{WO}_{3}, \sim 700^{\circ} \mathrm{C}$ for $\mathrm{MoO}_{3}$ and $150^{\circ} \mathrm{C}$ for sulfur. In the case of $\mathrm{MoS}_{2}$ flake growth, $150 \mathrm{mg}$ Sulphur and $1.5 \mathrm{mg} \mathrm{MoO}_{3}$ powder are used as precursors and likewise, for the case of $\mathrm{WS}_{2}, 150 \mathrm{mg} \mathrm{S}$ and $1.5 \mathrm{mg} \mathrm{WO}_{3}$ powder are used. Table 1 lists the process parameters optimized for $2 \mathrm{D} \mathrm{MoS} 2$ and $\mathrm{WS}_{2}$. Since radical vapor ratios are critical to obtain mono-layer formation, substrate temperature and their spatial locations are carefully adjusted. These parameters have been studied in our previous studies $[32,33] . \mathrm{D}_{\mathrm{s}}$ indicates the distance between boats of $\mathrm{MoO}_{3}$ or $\mathrm{WO}_{3}$ powder and the substrates in horizontal growth configuration; $\mathrm{T}_{\mathrm{S}}$ indicates the temperature of the substrate.

In addition to the planar growth, it is also possible to use face-down growth configuration as presented in the inset of Figure 1. In this configuration, $\mathrm{MoO}_{3}$ powder is placed in a quartz cylinder that has a height and a diameter of $2 \mathrm{~cm}$. The $2 \mathrm{~cm}^{2} \mathrm{Si} / \mathrm{SiO}_{2}$ substrate is placed on this cylinder and $\mathrm{MoS}_{2}$ is formed on the downward facet of the substrate at $700{ }^{\circ} \mathrm{C}$ by using $400 \mathrm{sccm} \mathrm{N} \mathrm{N}_{2}$ under atmospheric pressure. As shown in the inset of Figure 1, there is a $2 \mathrm{~cm}$ vertical difference between the position of the precursor and the substrate where the substrate is placed on the quartz boat. In our experiments, we used planar configuration for $2 \mathrm{D} \mathrm{WS} 2$ growth and face-down configuration for $2 \mathrm{D} \mathrm{MOS}_{2}$ growth. 
Regarding the deposition, just before process starts, the $\mathrm{SiO}_{2}(\sim 300 \mathrm{~nm})$ coated $\mathrm{Si}$ substrates are cleaned by piranha etch solution and RCA technique. The temperature of reaction chamber is increased at a rate of $15^{\circ} \mathrm{C} / \mathrm{min}$ using nitrogen gas. For the case of $\mathrm{WS}_{2}$ growth, $\mathrm{H}_{2}$ is also introduced to the system as a carrying gas to promote $2 \mathrm{D}$ formations. $\mathrm{H}_{2}$ is reported to enhance the size and density of crystals due to the increased reduction of $\mathrm{WO}_{3}$, however, excessive amount is stated to cause the grown $\mathrm{WS}_{2}$ films to be etched [35]. Hence, in this research, $5 \%$ of $\mathrm{H}_{2}$ is an optimized value for favoring the 2D $\mathrm{WS}_{2}$ growth. The distance between the precursors of sulphur and $\mathrm{MoO}_{3} / \mathrm{WO}_{3}$ is kept at $16 \mathrm{~cm}$ but the furnace is pulled $4 \mathrm{~cm}$ towards the sulphur powder to increase the sulphur vapor pressure as the growth temperature is reached.

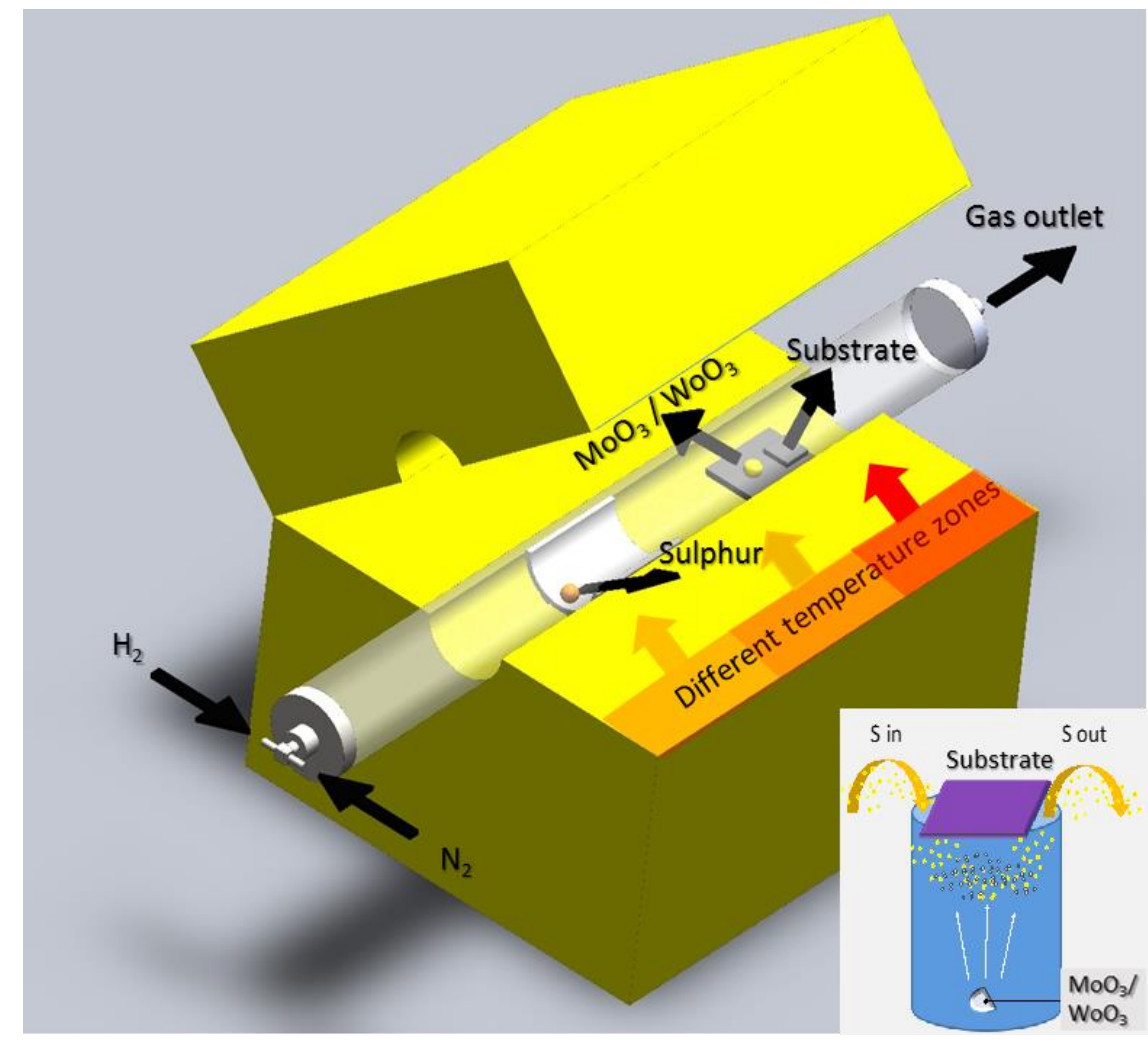

Figure 1. (a) The experimental setup of the CVD designed specifically for 2D TMDC growth with horizontal configuration. Substrate holder for the case of face-down growth is shown in the inset

Table 1. CVD process parameters for $2 \mathrm{D} \mathrm{MoS}_{2}$ and $\mathrm{WS}_{2}$ growth

\begin{tabular}{ccccccc}
\hline & $\mathbf{T}_{\mathbf{S}}\left({ }^{\circ} \mathbf{C}\right)$ & $\mathbf{D}_{\mathbf{S}}(\mathbf{c m})$ & $\begin{array}{c}\text { Growth duration } \\
(\text { minutes })\end{array}$ & $\begin{array}{c}\mathbf{N}_{2}-\text { Flow rate } \\
(\mathbf{s c c m})\end{array}$ & $\begin{array}{c}\mathbf{H}_{2}-\text { Flow rate } \\
(\mathbf{s c c m})\end{array}$ & Pressure \\
\hline $\mathrm{MoS}_{2}$ & data & $($ face-down) & 3 & 400 & - & ambient \\
$\mathrm{WS}_{2}$ & 820 & 1.5 & 3 & 380 & 20 & ambient \\
\hline
\end{tabular}

When the deposition temperature is reached, $\mathrm{S}$ vapor reduces $\mathrm{MoO}_{3} / \mathrm{WO}_{3}$ powder to volatile suboxides producing intermediate products such as $\mathrm{MoO}_{2}$ and $\mathrm{WO}_{2}$ [36] and the formed radicals diffuse on the substrate reacting with sulfur and monolayers are realized by the equations (1-2) and (3-4), for $\mathrm{MoS}_{2}$ and $\mathrm{WS}_{2}$, respectively $[23,37]$. In general, precursor ratio (in fact, the dissociated radicals' ratio) is suggested to be the essential internal parameter determining the monolayer formation. The optimal sulfurization process can be completed by controlling this ratio carefully. As the deposition duration is finished, a rapid cooling down process needs to be carried out to avoid other products such as $\mathrm{MoO}_{3}$ and multi layer growth of $\mathrm{MoS}_{2}$ where $500 \mathrm{sccm}$ of nitrogen gas (99.999\%) is purged in the tube. 


$$
\begin{gathered}
\mathrm{MoO}_{3}+(\mathrm{x} / 2) \mathrm{S} \rightarrow \mathrm{MoO}_{3-\mathrm{x}}+(\mathrm{x} / 2) \mathrm{SO}_{2} \\
\mathrm{MoO}_{3-\mathrm{x}}+(7-\mathrm{x}) / 2 \mathrm{~S} \rightarrow \mathrm{MoS}_{2}+(3-\mathrm{x}) / 2 \mathrm{SO}_{2} \\
7 \mathrm{~S}+2 \mathrm{WO}_{3} \rightarrow 2 \mathrm{WS}_{2}+3 \mathrm{SO}_{2} \\
\mathrm{WO}_{3}+3 \mathrm{~S}+\mathrm{H}_{2} \rightarrow \mathrm{WS}_{2}+\mathrm{SO}_{2}+\mathrm{H}_{2} \mathrm{O}
\end{gathered}
$$

We carried out Raman and PL spectral characterizations with Witec $\alpha$-300 R $\mu$-Raman system at room temperature where we used $532 \mathrm{~nm}$ excitation wavelength for both Raman and PL measurements. We obtained the images of the flakes by confocal optical microscopy and differential interference contrast (DIC) microscopy with Nikon Eclipse LV100NDA microscope. DIC is utilized to enhance the contrast in our monolayers, which are inherently transparent. The technique is based on the principle of interferometry providing the optical path length of the sample allowing us to see the features such as grain boundaries, which are otherwise invisible [38].

\section{RESULTS and DISCUSSION}

Figure 2 (a) shows the images of the $\mathrm{MoS}_{2}$ flake images obtained measured by confocal optical microscopy. As a result of the described growth procedure, flakes with a side of $40 \mu \mathrm{m}$ are formed. In this growth scheme, we observe that flakes touch, overlap or merge to form larger. In general, with this configuration, we obtain flakes that are radially distributed over the substrate. Center of the substrate contains rather smaller flakes whereas these flakes get larger when we get closer to the edges of the substrate. The size of the flakes can be adjusted by changing the duration of positioning the furnace to the left hand side (onto the sulphur) when the growth zone reaches $700{ }^{\circ} \mathrm{C}$. This change in flake size is attributed to the slower sulfurization process. It is reported that the film formation occurs in TMDCs by the growth of individual flakes and their coalescence or merging $[39,40]$. Therefore, some extra growth time initiate a slower growth process (little $\mathrm{S}$ vapor can reach the substrate) and the flakes grow larger till they merge to form a continuous monolayer film.

The designated region with a red rectangle on the image region is also investigated by dark field optical microscopy (Figure 2 (b)). Grain-boundary structure, which is not visible by optical microscopy but visible in the dark field optical images in Figure 2 (b) suggests that the flakes are not epitaxially connected but rather overlapped. This can be attributed to the amorphous $\mathrm{SiO}_{2}$ layer that does not support van der Waals epitaxy as lattice matched $\mathrm{c}-\mathrm{Al}_{2} \mathrm{O}_{3}$ and $\mathrm{GaN}$ would provide $[41,42]$. The orange arrows point out to the boundaries inside the flake (rather straight lines) and the green arrow indicates the seed. For the $\mathrm{MoS}_{2}$ flakes to grow these seeds are needed, hence a large number of seed cause smaller flakes.

Figure 2 (c) shows the Raman scattering spectra between 360 and $430 \mathrm{~cm}^{-1}$. MoS 2 has two characteristic Raman peaks corresponding to in-plane vibration of Mo and $S$ atoms $\left(E_{2 \mathrm{~g}}^{1}\right)$ at $383 \mathrm{~cm}^{-1}$ and the out-ofplane vibration of $\mathrm{S}$ atoms $\left(\mathrm{A}_{\mathrm{g}}^{1}\right)$ at $408 \mathrm{~cm}^{-1}$ where the change in difference between these two peaks is used as an indicator for the number of layers [41]. When the layers decrease, the mode at $383 \mathrm{~cm}^{-1}$ shifts to the lower frequencies and the mode at $408 \mathrm{~cm}^{-1}$ shifts to higher frequencies, [22, 39], resulting in closer positioned peaks. Figure 2 (d) presents delta Raman mapping image (difference between $A_{1 g}$ and $\mathrm{E}^{1}{ }_{2 \mathrm{~g}}$ peak positions). Raman single spectra in Figure 2 (c) are taken from the points shown by the arrows on this map (red: P1 and green P2). Boundaries inside the flakes indicated by orange arrows in Figure 2 (b) point to the existence of structural defects (line defects). SNOM-PL-analysis and SEM investigation 
of butterfly like flakes reveal $20 \mathrm{~nm}$ wide line defects inside the flake [43]. These line defects are labeled as grain-boundaries inside the flake due to the growth nature of the butterfly shape where more than one flake seem to merge [43]. However, in our case, the line defects demonstrated in Figure 2 (b) inside the single flake are hardly found to be the grain-boundary but more likely to be the defects related to the stress effect [44]. On the other hand, green arrow pointing the brighter dot indicates the seed initiating the growth of the flakes [45]. Despite the overlapped regions, flakes that have no contact with their neighbor are monolayers. This can be evidenced from the Figure 2 (c). In general, this difference depends on the preparation method of the $\mathrm{MoS}_{2}$ flakes. For example, mechanically exfoliated $\mathrm{MoS}_{2}$ flakes have $18 \mathrm{~cm}^{-1}$ Raman peak difference [46]. On the other hand, the difference in CVD grown flakes might have higher values such as $22 \mathrm{~cm}^{-1}$ for a single- layer flake [47], which agrees with our delta map. In our delta map, in general, we observe a delta of $\sim 22 \mathrm{~cm}^{-1}$ as shown in Figure 2 (c). Maximum peak difference is observed in those reddish data and they never exceed above $22.2 \mathrm{~cm}^{-1}$ as demonstrated in Figure 2(d).

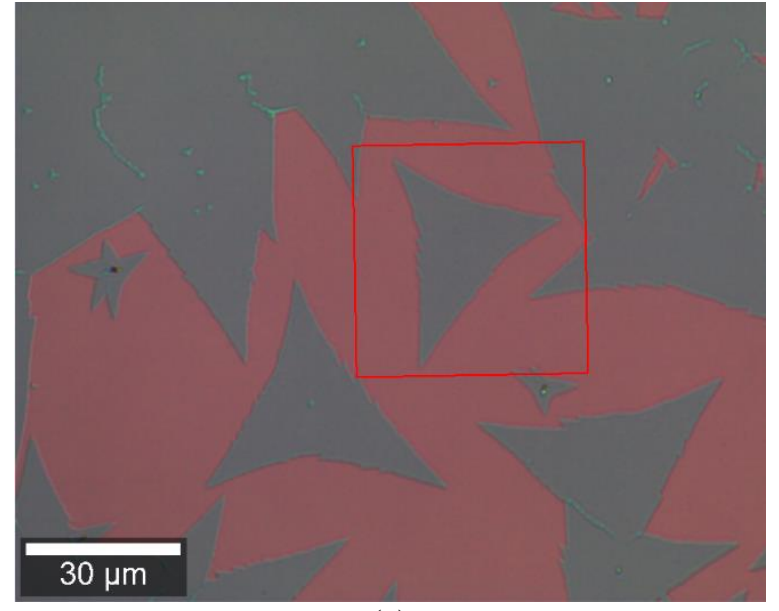

(a)

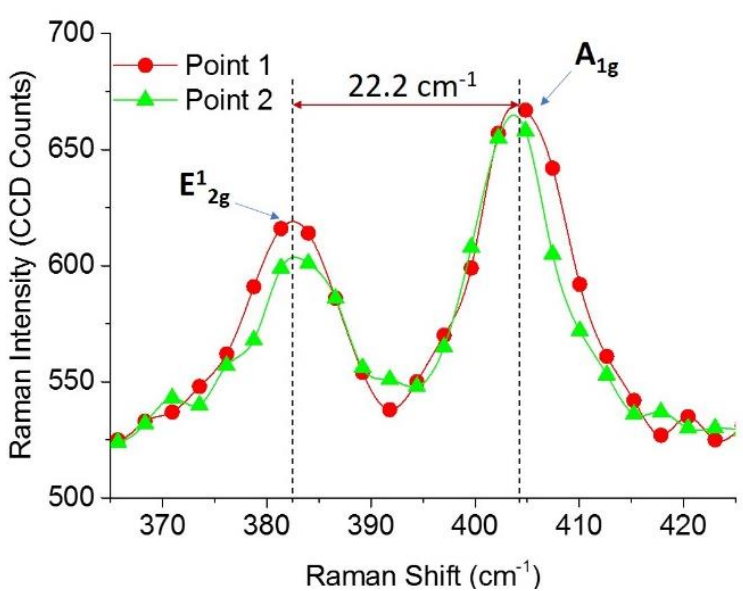

(c)

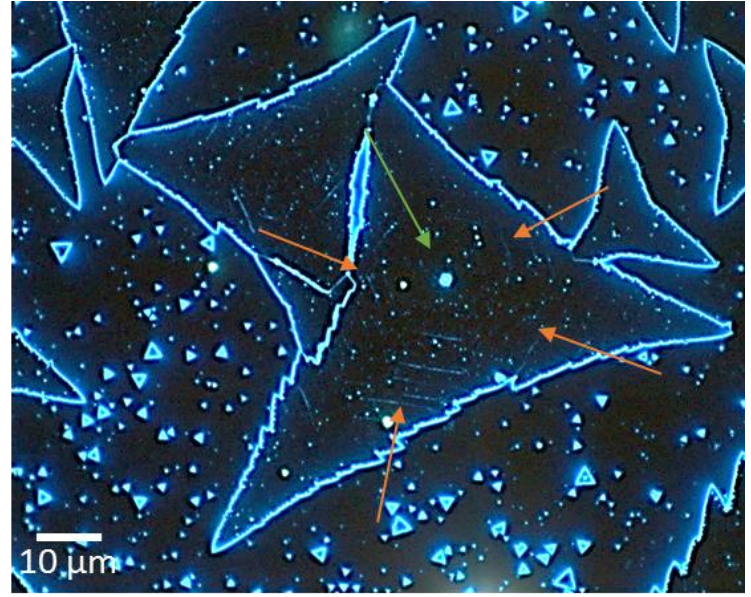

(b)

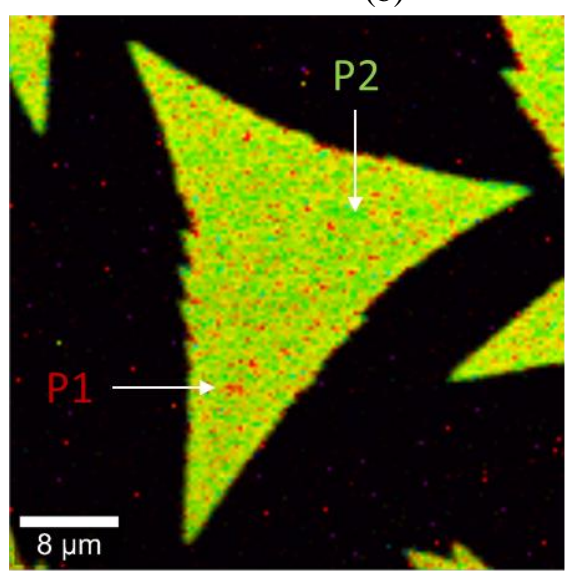

$23 \mathrm{~cm}^{-1}$

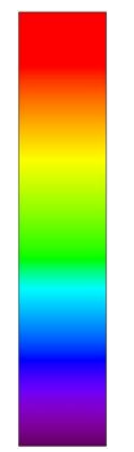

$18 \mathrm{~cm}^{-1}$

(d)

Figure 2. a) Confocal optical image of $\mathrm{MoS}_{2}$ flakes. Analyzed region is demonstrated with a red rectangle on the image b) Dark field optical microscope image of $\mathrm{MoS}_{2}$. Orange arrows point straight lines inside the flake and green arrow points the seed c) Raman scattering spectra d) delta Raman mapping image (difference between $\mathrm{E}^{1}{ }_{2 \mathrm{~g}}$ and $\mathrm{A}_{\mathrm{g}}^{1}$ peaks); the points demonstrated by arrows on the map are the positions where Raman spectra is measured. 
Realization of indirect to direct electronic band gap transition and the resulting high-intensity PL has appealed substantial attention to study optical properties of such large-area monolayers aiming for novel photonic devices [48]. Figure 3 exhibits the photoluminescence properties of the grown $\mathrm{MoS}_{2}$ monolayers. $45 \times 45 \mu \mathrm{m}^{2}$ area with $150 \times 150$ data points (22500 data points in total) are scanned with a 532 $\mathrm{nm}$ laser, $1 \mathrm{~mW}$ laser power and 1 second integration time. Figure 3(a) demonstrates the total PL intensity map. It is obtained by calculating the total area under the PL curve for each data points. Since the value of the area is not a convenient measure to construct a colored scale bar, the total PL intensity is normalized according maximum area value to get a normalized total intensity map. Red zones in this map therefore, demonstrates the regions where maximum PL intensity is obtained. From the figure, it is seen that the PL intensity is not fully uniform where PL intensity reduces at rather sharp edges of the triangular flake.

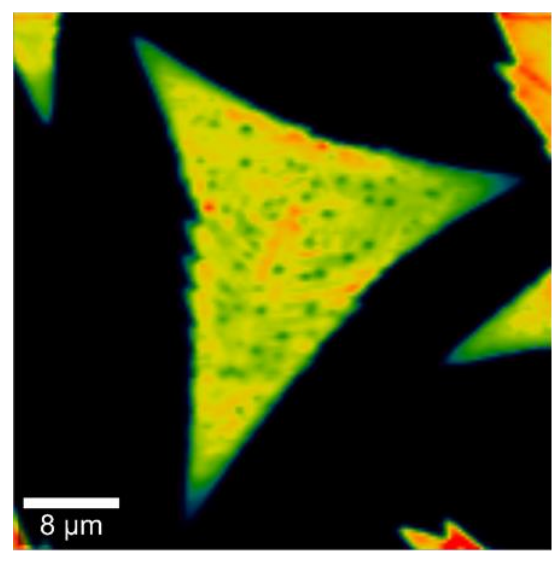

(a)

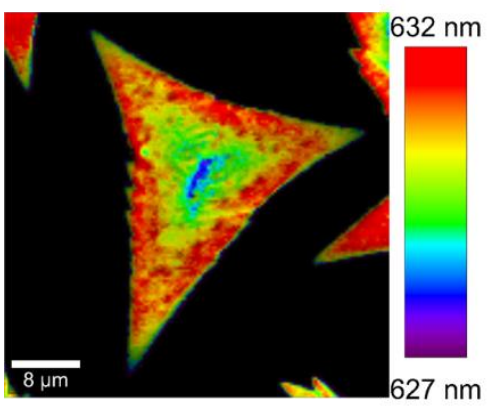

(c)
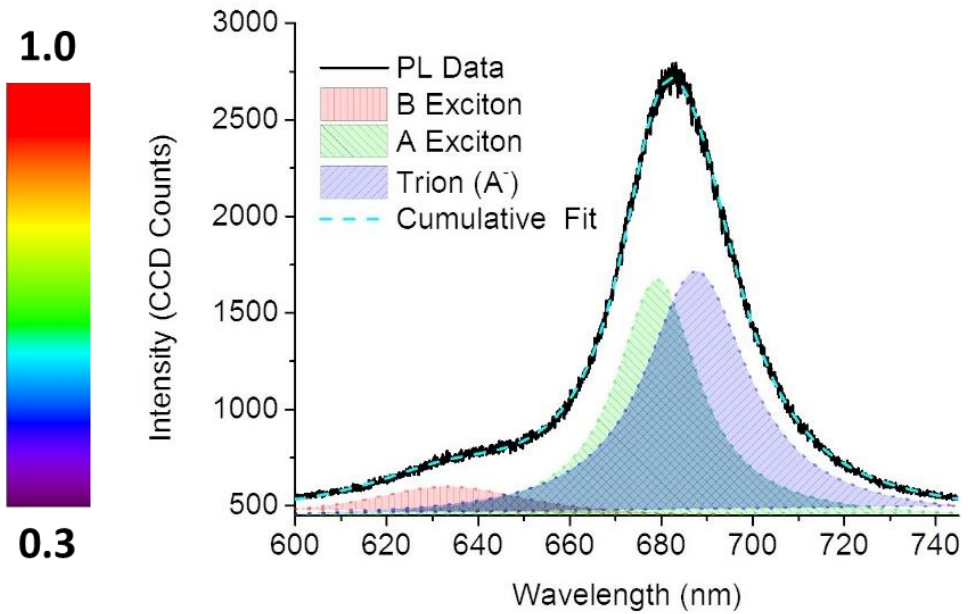

(b)

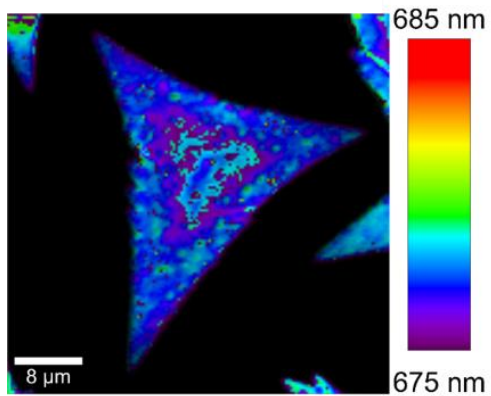

(d)

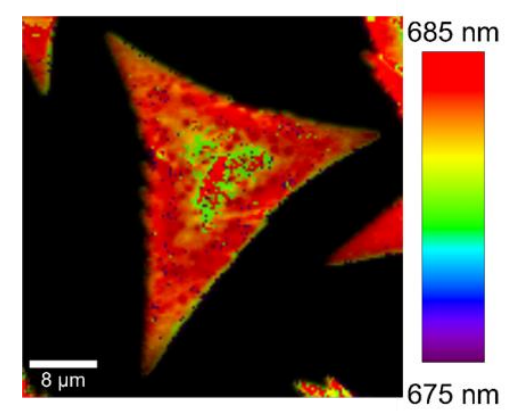

(e)

Figure 3. a) Integrated and normalized total PL intensity b) single spectrum of a random point taken from the flake and the fitted curves c) B exciton peak center map d) A exciton peak center map e) Trion peak center map

PL spectrum in Figure 3(b) can be deconvoluted into three Lorentzian peaks similar to the cases studied in different reports [49-51]. These deconvoluted peaks can be further be used to study the PL phenomena in these materials. Physical interpretation of different peaks is related with the exciton and trion generation and recombination. The B exciton peak between $627 \mathrm{~nm}$ to $632 \mathrm{~nm}$ (Figure 2(c)), A exciton peak between $675 \mathrm{~nm}$ to $678 \mathrm{~nm}$ (Figure 3(d)), and trion (A) peak between $680 \mathrm{~nm}$ and $685 \mathrm{~nm}$ (Figure 3(d)) are mapped for the same flake. Different energy levels of A and B peaks are attributed to the valance band splitting at $\mathrm{K}$ point due to the strong spin-orbit coupling inherent to the TMDC materials. It is interesting to note that the PL peak center of the A, B and $\mathrm{A}^{-}$shifts according to flake position, which accompanies with the intensity variation. By using the intensity data of the $\mathrm{A}$ and $\mathrm{A}^{-}$from the fitted curves, we may map the major photoluminescence mechanism. In order to accomplish this, the 
intensity ratio of the $\mathrm{A}$ and $\mathrm{A}^{-}$is calculated and the resulting map image is demonstrated in Figure 4. The map depicts that the majority of the edges are slightly dominated by the trion PL mechanism (A/A is just below 1). A small fraction at the center zone (where possibly the seed resides) is dominated by the A exciton.

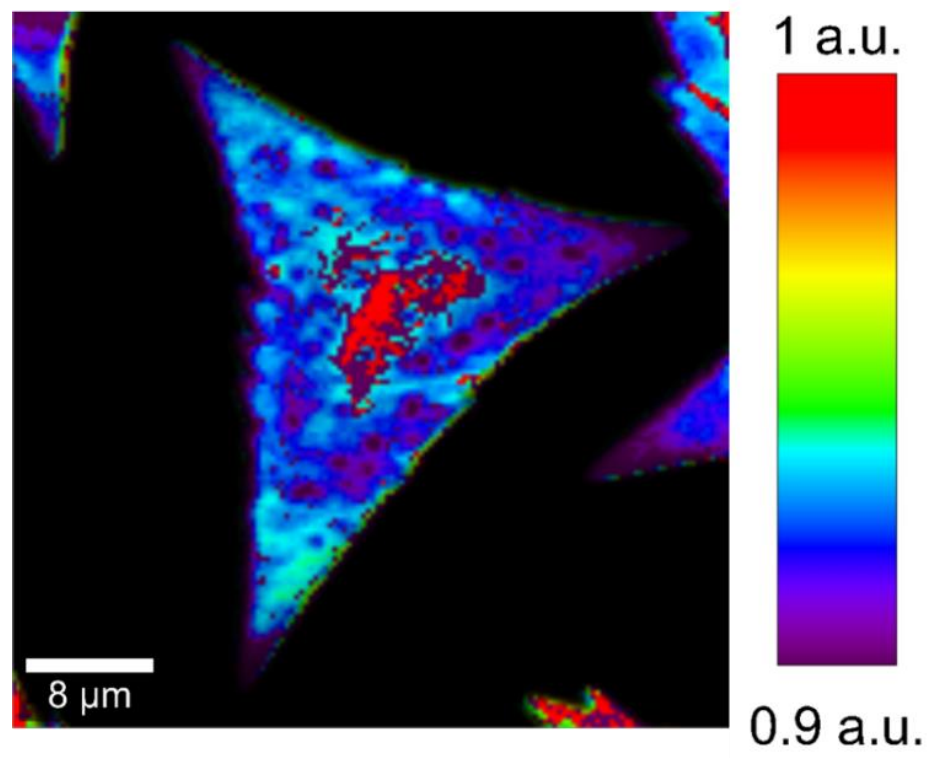

(a)

Figure 4. Map of intensity ratio of A exciton to trion luminescence

Figure 5 (a) shows optical microscopy images of $2 \mathrm{D} \mathrm{WS}_{2}$ flakes. In Figure 5(b) the same region is presented by the DIC microscopy where grain-boundaries are more visible similar to the $\mathrm{MoS}_{2}$ case. Likewise the $\mathrm{WS}_{2}$ flakes are overlapped. Also, for the $\mathrm{WS}_{2}$ flakes, seeds are located at the center of the flakes, which indicates that for large and uniform film growth, these seeds need to be carefully controlled.

Figure 5 (c) shows the Raman scattering spectra between 280 and $380 \mathrm{~cm}^{-1}$. Presented Raman modes in Figure 5(c) are in agreement with the previously reported main Raman peaks, which are $\mathrm{E}_{2 G}^{1}(\Gamma)$ and $\mathrm{A}_{1 G}(\Gamma)$ at $\sim 350 \mathrm{~cm}^{-1}$ and $\sim 415 \mathrm{~cm}^{-1}$ respectively. It is stated that $62.2 \mathrm{~cm}^{-1}$ frequency difference between these two peaks is accepted as an indicator of monolayer $\mathrm{WS}_{2}$ structure at $532 \mathrm{~nm}$ laser excitation wavelength $[52,53]$. In addition to that, as it is reported by some studies, the peak between $\sim 295 \mathrm{~cm}^{-1}$ and $\sim 325 \mathrm{~cm}^{-1}$ which is at $\sim 310 \mathrm{~cm}^{-1}$ tends to disappear when $\mathrm{WS}_{2}$ transits from a few layers to a monolayer[54], which is also the case for our as-grown $\mathrm{WS}_{2}$ flakes are monolayers. 


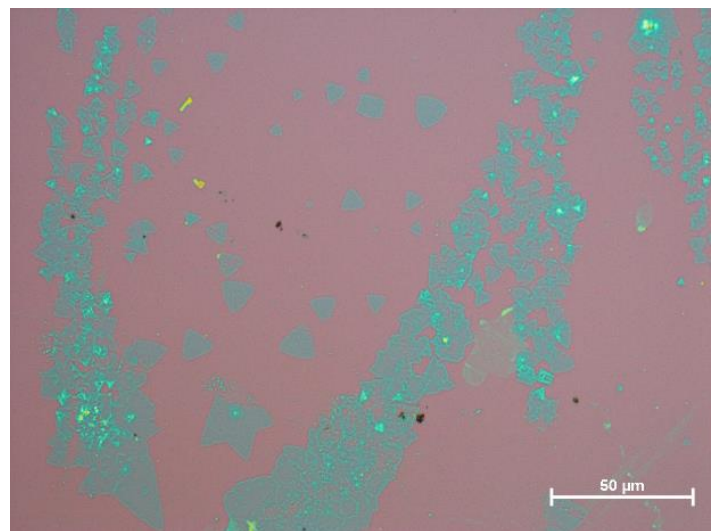

(a)

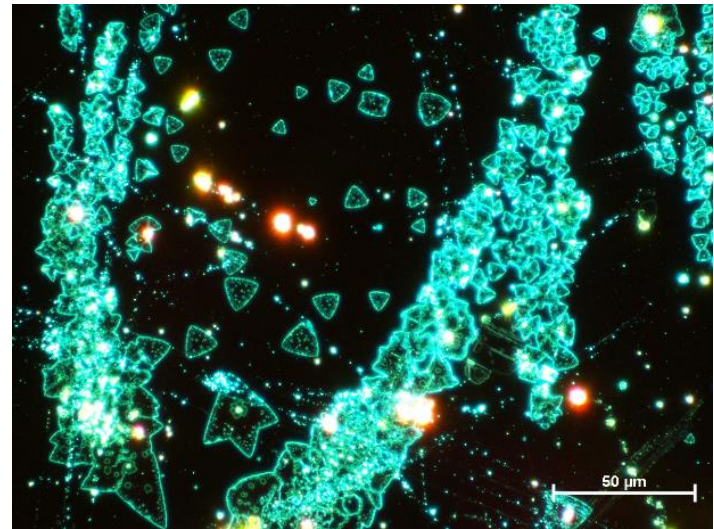

(b)

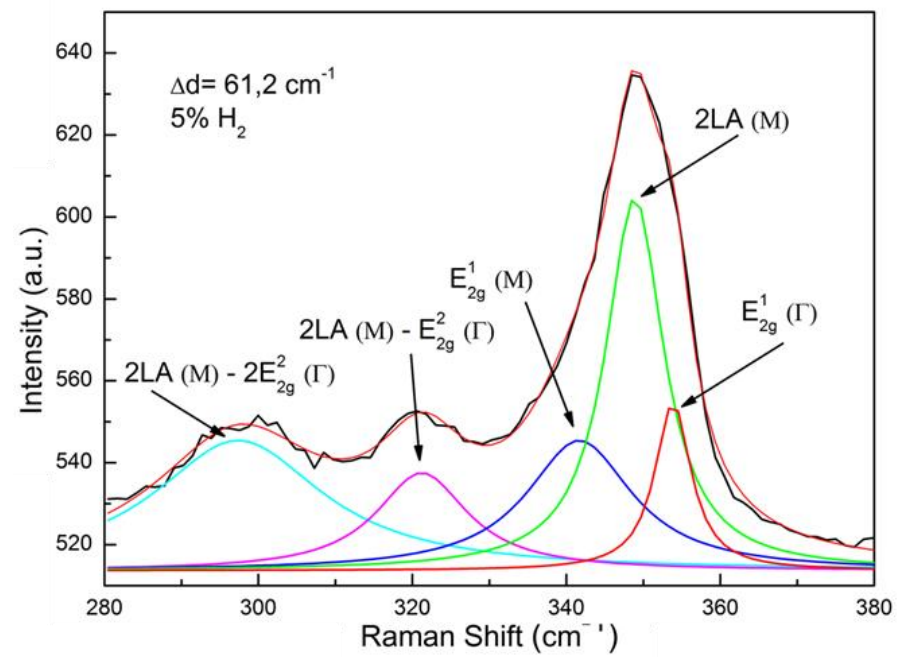

(c)

Figure 5. a) Confocal optical image of $\mathrm{WS}_{2}$ flakes b) the same region shown by dark field optical microscopy c) Raman scattering spectra

To have further understanding of the optical and structural properties of $\mathrm{WS}_{2}$ formations, one of the flakes of which the optical microscopy image is shown in Figure 6 (a) is investigated through its photoluminescence properties. Figure 6 (b) shows the PL spectra with its deconvoluted peaks; the exciton peak at $\sim 637 \mathrm{~nm}$ and trion peak at $\sim 650 \mathrm{~nm}[34,55]$. In Figure 6 (c), maximum PL intensity map contains dark regions where we do not observe PL. These dark regions are suggested to be the seeds. Although for the 2D TMDC formation these seeds are needed, under our growth conditions, the seeding process continues after the flake is grown, which causes multi-layer film growth. Hence, PL intensity mapping also provides us to clarify the homogeneity of the flakes which is not possible by only confocal optical microscopy. In addition to these seeds and partial multi-layered structure of the flake, there is shift in the PL central wavelength as shown in Figure 6 (d). This result shows that luminescence is due to trion emission at the edges whereas excitonic emission is more profound at the interior regions of the flake. 


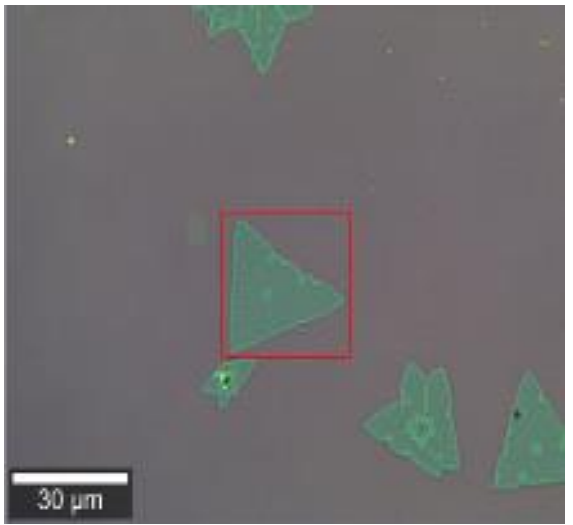

(a)

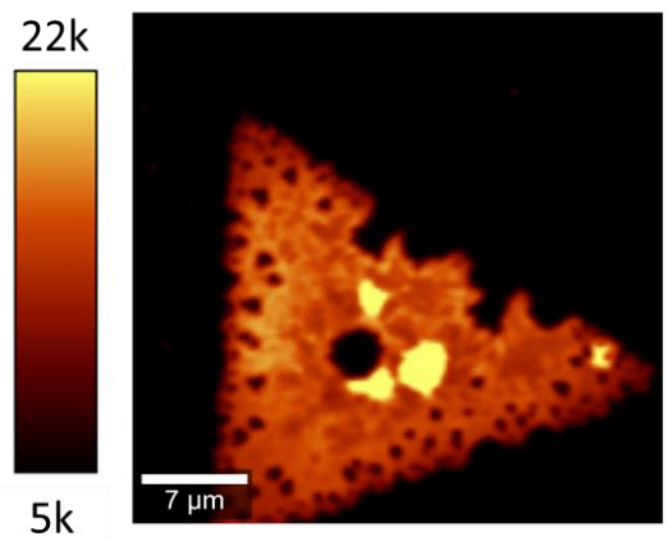

(c)

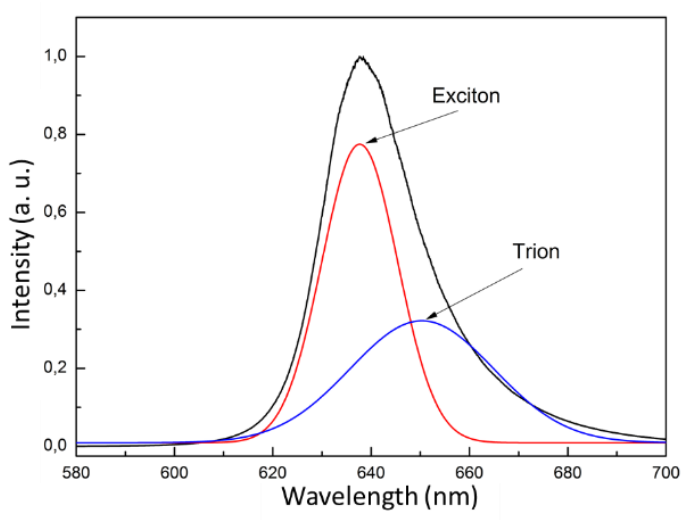

(b)

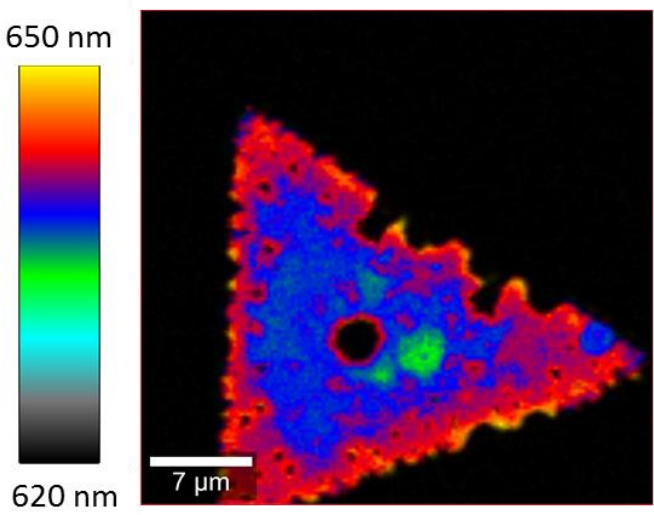

(d)

Figure 6. For the same $\mathrm{WS}_{2}$ flake (a) confocal optical image (b) PL spectra (c) maximum PL intensity map (d) PL peak center map

\section{CONCLUSIONS}

By using the CVD technique, we obtained $\mathrm{MoS}_{2}$ and $\mathrm{WS}_{2}$ monolayers larger than $30 \mu \mathrm{m}$. These results confirm that CVD is a powerful technique to control and tune the properties of the monolayer flakes. Since the structural and optical characteristic of these TMDC material systems is a matter of research, we first measured $\mu$ Raman and photoluminescence spectra and we performed dark field microscopy measurements together with photoluminescence mappings so that grain boundaries and seeding particles could be identified. Detailed analysis of PL spectra maps reveal that emission occurs due to different mechanisms such as excitons and trions where the dominant mechanism differs depending on the spatial locations of the flakes. DIC images are also in agreement with the PL maps regarding the grain boundaries and seeds exposing that dark field imaging can be used a rapid and reliable technique for such an analysis.

\section{ACKNOWLEDGEMENTS}

This work was supported by Anadolu University Research Project no: BAP1605F424 and BAP1407F335. 
Kosku Perkgöz / Anadolu Univ. J. of Sci. and Technology A-Appl. Sci. and Eng. 18 (2) - 2017

\section{REFERENCES}

[1] Nalwa HS. Handbook of Advanced Electronic and Photonic Materials and Devices: Semiconductors. Vol. 1: Academic Press; 2001.

[2] Bogaert K, Liu S, Chesin J, Titow D, Gradečak S, Garaj S. Diffusion-Mediated Synthesis of MoS2/WS2 Lateral Heterostructures. Nano Letters. 2016;16:5129-34.

[3] Xia FN, Yan HG, Avouris P. The Interaction of Light and Graphene: Basics, Devices, and Applications. Proceedings of the Ieee. 2013;101:1717-31.

[4] Zhu Y, Murali S, Cai W, Li X, Suk JW, Potts JR, et al. Graphene and graphene oxide: synthesis, properties, and applications. Advanced Materials. 2010;22:3906-24.

[5] Geim AK. Graphene: status and prospects. Science. 2009;324:1530-4.

[6] Bonaccorso F, Sun Z, Hasan T, Ferrari AC. Graphene photonics and optoelectronics. Nature Photonics. 2010;4:611-22.

[7] Pop E, Varshney V, Roy AK. Thermal properties of graphene: Fundamentals and applications. Mrs Bulletin. 2012;37:1273-81.

[8] Zhi Y, Rungang G, Nantao H, Jing C, Yingwu C, Liying Z, et al. The Prospective 2D Graphene Nanosheets: Preparation, Functionalization and Applications. Nano-Micro Letters. 2012;4:1-9.

[9] Gibney E. The super materials that could trump graphene. Nature. 2015;522.

[10] Bonaccorso F, Lombardo A, Hasan T, Sun Z, Colombo L, Ferrari AC. Production and processing of graphene and 2d crystals. Materials Today. 2012;15:564-89.

[11] Xiang Q, Yu J, Jaroniec M. Synergetic Effect of MoS2 and Graphene as Cocatalysts for Enhanced Photocatalytic H2 Production Activity of TiO2 Nanoparticles. Journal of the American Chemical Society. 2012;134:6575-8.

[12] Komsa H-P, Krasheninnikov AV. Two-dimensional transition metal dichalcogenide alloys: stability and electronic properties. The Journal of Physical Chemistry Letters. 2012;3:3652-6.

[13] Berkelbach TC, Hybertsen MS, Reichman DR. Theory of neutral and charged excitons in monolayer transition metal dichalcogenides. Physical Review B. 2013;88:045318.

[14] Wang QH, Kalantar-Zadeh K, Kis A, Coleman JN, Strano MS. Electronics and optoelectronics of two-dimensional transition metal dichalcogenides. Nat Nano. 2012;7:699-712.

[15] Ji Q, Zhang Y, Gao T, Zhang Y, Ma D, Liu M, et al. Epitaxial Monolayer MoS2 on Mica with Novel Photoluminescence. Nano Letters. 2013;13:3870-7.

[16] Zeng H, Dai J, Yao W, Xiao D, Cui X. Valley polarization in MoS2 monolayers by optical pumping. Nat Nano. 2012;7:490-3.

[17] Shi J, Ma D, Han G-F, Zhang Y, Ji Q, Gao T, et al. Controllable Growth and Transfer of Monolayer MoS2 on Au Foils and Its Potential Application in Hydrogen Evolution Reaction. ACS nano. 2014. 
[18] Xin T, Eric A, Feng L, Handong L, Zhiming MW. Advances in MoS2-based Field Effect Transistors (FETs). Nano-Micro Letters. 2015;7.

[19] Chang K, Chen W. L-cysteine-assisted synthesis of layered MoS2/graphene composites with excellent electrochemical performances for lithium ion batteries. ACS nano. 2011;5:4720-8.

[20] Cong C, Shang J, Wu X, Cao B, Peimyoo N, Qiu C, et al. Synthesis and Optical Properties of Large-Area Single-Crystalline 2D Semiconductor WS2 Monolayer from Chemical Vapor Deposition. Advanced Optical Materials. 2014;2:131-6.

[21] Mak KF, Lee C, Hone J, Shan J, Heinz TF. Atomically thin $\mathrm{MoS}_{2}$ : a new direct-gap semiconductor. Physical Review Letters. 2010;105:136805.

[22] Chen H, Wen X, Zhang J, Wu T, Gong Y, Zhang X, et al. Ultrafast formation of interlayer hot excitons in atomically thin MoS2/WS2 heterostructures. Nature Communications. 2016;7:12512.

[23] Lee Y-H, Zhang X-Q, Zhang W, Chang M-T, Lin C-T, Chang K-D, et al. Synthesis of Large-Area MoS2 Atomic Layers with Chemical Vapor Deposition. Advanced Materials. 2012;24:2320-5.

[24] Zhan Y, Liu Z, Najmaei S, Ajayan PM, Lou J. Large - Area Vapor - Phase Growth and Characterization of $\mathrm{MoS}_{2}$ Atomic Layers on a SiO2 Substrate. Small. 2012;8:966-71.

[25] Coleman JN, Lotya M, O’Neill A, Bergin SD, King PJ, Khan U, et al. Two-dimensional nanosheets produced by liquid exfoliation of layered materials. Science. 2011;331:568-71.

[26] van der Zande AM, Huang PY, Chenet DA, Berkelbach TC, You Y, Lee G-H, et al. Grains and grain boundaries in highly crystalline monolayer molybdenum disulphide. Nature Materials. 2013;12:554-61.

[27] Liu K-K, Zhang W, Lee Y-H, Lin Y-C, Chang M-T, Su C-Y, et al. Growth of Large-Area and Highly Crystalline MoS2 Thin Layers on Insulating Substrates. Nano Letters. 2012;12:1538-44.

[28] Zhan Y, Liu Z, Najmaei S, Ajayan PM, Lou J. Large-Area Vapor-Phase Growth and Characterization of MoS2 Atomic Layers on a SiO2 Substrate. Small. 2012;8:966-71.

[29] Kim IS, Sangwan VK, Jariwala D, Wood JD, Park S, Chen K-S, et al. Influence of Stoichiometry on the Optical and Electrical Properties of Chemical Vapor Deposition Derived MoS2. ACS Nano. 2014.

[30] Zhang J, Yu H, Chen W, Tian X, Liu D, Cheng M, et al. Scalable Growth of High-Quality Polycrystalline MoS2 Monolayers on SiO2 with Tunable Grain Sizes. ACS Nano. 2014;8:6024-30.

[31] Wang S, Rong Y, Fan Y, Pacios M, Bhaskaran H, He K, et al. Shape Evolution of Monolayer MoS2 Crystals Grown by Chemical Vapor Deposition. Chemistry of Materials. 2014;26:6371-9.

[32] Perkgoz NK, Bay M. Investigation of Single-Wall MoS2 Monolayer Flakes Grown by Chemical Vapor Deposition. Nano-Micro Letters. 2016;8:70-9.

[33] Özden A, Şar H, Yeltik A, Madenoğlu B, Sevik C, Ay F, et al. CVD grown 2D MoS2 layers: A photoluminescence and fluorescence lifetime imaging study. physica status solidi (RRL)-Rapid Research Letters. 2016;10:792-6. 
Kosku Perkgöz / Anadolu Univ. J. of Sci. and Technology A - Appl. Sci. and Eng. 18 (2) - 2017

[34] Kim MS, Yun SJ, Lee Y, Seo C, Han GH, Kim KK, et al. Biexciton emission from edges and grain boundaries of triangular ws2 monolayers. ACS Nano. 2016;10:2399-405.

[35] Kang KN, Godin K, Yang E-H. The growth scale and kinetics of WS2 monolayers under varying H2 concentration. Scientific reports. 2015;5.

[36] Li XL, Li YD. Formation of MoS2 Inorganic Fullerenes (IFs) by the Reaction of MoO3 Nanobelts and S. Chemistry - A European Journal. 2003;9:2726-31.

[37] Zhang Y, Zhang Y, Ji Q, Ju J, Yuan H, Shi J, et al. Controlled growth of high-quality monolayer WS2 layers on sapphire and imaging its grain boundary. ACS Nano. 2013;7:8963-71.

[38] Lang W. Nomarski differential interference-contrast microscopy: Oberkochen, Carl Zeiss; 1982.

[39] Chen CY, Qiao H, Xue YZ, Yu WZ, Song JC, Lu Y, et al. Growth of large-area atomically thin MoS2 film via ambient pressure chemical vapor deposition. Photonics Research. 2015;3:110-4.

[40] Najmaei S, Liu Z, Zhou W, Zou X, Shi G, Lei S, et al. Vapour phase growth and grain boundary structure of molybdenum disulphide atomic layers. Nat Mater. 2013;12:754-9.

[41] Dumcenco D, Ovchinnikov D, Marinov K, Lazic P, Gibertini M, Marzari N, et al. Large-Area Epitaxial Mono layer MoS2. Acs Nano. 2015;9:4611-20.

[42] Ruzmetov D, Zhang K, Stan G, Kalanyan B, Bhimanapati GR, Eichfeld SM, et al. Vertical 2D/3D Semiconductor Heterostructures Based on Epitaxial Molybdenum Disulfide and Gallium Nitride. Acs Nano. 2016;10:3580-8.

[43] Lee Y, Park S, Kim H, Han GH, Lee YH, Kim J. Characterization of the structural defects in CVDgrown monolayered MoS2 using near-field photoluminescence imaging. Nanoscale. 2015;7:11909-14.

[44] Özden A, Şar H, Yeltik A, Madenoğlu B, Sevik C, Ay F, et al. CVD grown 2D MoS 2 layers: A photoluminescence and fluorescence lifetime imaging study. physica status solidi (RRL) - Rapid Research Letters. 2016;10:792-6.

[45] Cain JD, Shi F, Wu J, Dravid VP. Growth Mechanism of Transition Metal Dichalcogenide Monolayers: The Role of Self-Seeding Fullerene Nuclei. Acs Nano. 2016;10:5440-5.

[46] Li H, Zhang Q, Yap CCR, Tay BK, Edwin THT, Olivier A, et al. From bulk to monolayer MoS2: evolution of Raman scattering. Advanced Functional Materials. 2012;22:1385-90.

[47] Jeon J, Jang SK, Jeon SM, Yoo G, Jang YH, Park JH, et al. Layer-controlled CVD growth of largearea two-dimensional MoS2 films. Nanoscale. 2015;7:1688-95.

[48] Conley HJ, Wang B, Ziegler JI, Haglund Jr RF, Pantelides ST, Bolotin KI. Bandgap engineering of strained monolayer and bilayer MoS2. Nano Letters. 2013;13:3626-30.

[49] Mak KF, He K, Lee C, Lee GH, Hone J, Heinz TF, et al. Tightly bound trions in monolayer MoS2. Nat Mater. 2013;12:207-11.

[50] Lin Y, Ling X, Yu L, Huang S, Hsu AL, Lee Y-H, et al. Dielectric Screening of Excitons and Trions in Single-Layer MoS2. Nano Letters. 2014;14:5569-76. 
Kosku Perkgöz / Anadolu Univ. J. of Sci. and Technology A - Appl. Sci. and Eng. 18 (2) - 2017

[51] Yong X, Zhan W, Yongjie Z, Peng Z, Ruixue W, Teng J, et al. Controllable growth of monolayer MoS 2 by chemical vapor deposition via close $\mathrm{MoO} 2$ precursor for electrical and optical applications. Nanotechnology. 2017;28:084001.

[52] Peimyoo N, Shang J, Yang W, Wang Y, Cong C, Yu T. Thermal conductivity determination of suspended mono-and bilayer WS2 by Raman spectroscopy. Nano Research. 2015;8:1210-21.

[53] Zhao W, Ghorannevis Z, Chu L, Toh M, Kloc C, Tan P-H, et al. Evolution of electronic structure in atomically thin sheets of WS2 and WSe2. ACS Nano. 2012;7:791-7.

[54] Zhao W, Ghorannevis Z, Amara KK, Pang JR, Toh M, Zhang X, et al. Lattice dynamics in monoand few-layer sheets of WS 2 and WSe 2. Nanoscale. 2013;5:9677-83.

[55] Wei K, Liu Y, Yang H, Cheng X, Jiang T. Large range modification of exciton species in monolayer WS2. Applied Optics. 2016;55:6251-5. 